\title{
Fournier's gangrene during lenvatinib treatment: A case report
}

\author{
MARTINA BARONE $^{1}$, GIORGIO GRANI ${ }^{1}$, VALERIA RAMUNDO ${ }^{1}$, \\ TIZIANA GARRITANO ${ }^{2}$, COSIMO DURANTE $^{1}$ and ROSA FALCONE ${ }^{1}$ \\ ${ }^{1}$ Department of Translational and Precision Medicine, Sapienza University of Rome, I-00185 Rome; \\ ${ }^{2}$ Department of Surgical Sciences, Sapienza University of Rome, I-00161 Rome, Italy
}

Received July 31, 2019; Accepted February 21, 2020

DOI: $10.3892 / \mathrm{mco} .2020 .2031$

\begin{abstract}
Fournier's gangrene is a rare and severe complication reported in patients with cancer treated with antiangiogenic drugs, most frequently with bevacizumab. The present report describes the case of an 80-year-old man with radioactive iodine-refractory metastatic thyroid cancer treated with lenvatinib (an oral multikinase inhibitor with antiangiogenic properties) who developed Fournier's gangrene in the absence of other known risk factors. To the best of our knowledge, this is the first case described during treatment with lenvatinib. The condition was likely due to a perturbation of vascular endothelial cells of the skin due to the inhibition of VEGF/VEGFR signaling. Fournier's gangrene may be a class effect of antiangiogenic treatment that clinicians should be aware of, as early diagnosis and treatment are associated with an improved outcome.
\end{abstract}

\section{Introduction}

Lenvatinib is a multireceptor tyrosine kinase inhibitor with versatile application approved by the Food and Drug Administration (FDA), at different dose, for the treatment of locally recurrent or metastatic progressive radioactive iodine-refractory (RAIR) differentiated thyroid cancer (TC) (1), as well as renal cell carcinoma (in combination with everolimus) and hepatocellular carcinoma $(2,3)$. Lenvatinib inhibits several tyrosine kinase receptors implicated in pathogenic angiogenesis, tumor growth, and cancer progression, in addition to their normal cellular functions including the vascular endothelial growth factor (VEGF) receptors VEGFR1 (FLT1), VEGFR2 (KDR), and VEGFR3 (FLT4), the fibroblast growth factor receptors (FGFR) 1, 2, 3, and 4, the platelet-derived growth factor receptor alpha, KIT, and RET (4). In TC, the starting dose is $24 \mathrm{mg}$ daily (5). However,

Correspondence to: Dr Rosa Falcone, Department of Translational and Precision Medicine, Sapienza University of Rome, Viale dell' Università 37, I-00185 Rome, Italy

E-mail: rosa.falcone@uniroma1.it

Key words: Fournier's gangrene, antiangiogenic treatment, lenvatinib, toxicity it is possible, in clinical practice, consider starting at other dose (20, 14, $10 \mathrm{mg} /$ day and escalate or de-escalate as tolerated), based on clinical consideration (age, concomitant diseases, performance status and risk of adverse events) (6).

In recent years, antiangiogenic drugs, including monoclonal antibodies like bevacizumab and multikinase inhibitors like lenvatinib, have represented an enormous improvement in the treatment of several types of cancer and a shift towards personalized and precision therapy. However, they present some side effects that have never been observed with conventional chemotherapy. In clinical practice, the most frequent adverse events related to antiangiogenic drugs are cardiovascular and gastrointestinal problems, thromboembolic events, and dermatologic alterations including wound healing complications $(7,8)$.

We present the case of an 80-year-old man with stage IV TC diagnosed with Fournier's gangrene (FG) that was most likely related to the antiangiogenic effect of lenvatinib. Written informed consent was provided by the patient for the publication of his clinical information and images.

\section{Case report}

In January 2018, an 80-year-old man was admitted to the Department of Emergency due to an anorectal abscess. His past medical history was significant for hypertension, prostatic hyperplasia, and stage IV RAIR TC, with metastases to the lungs, mediastinum, and liver. He was not a smoker nor a drinker. He underwent total thyroidectomy in 2000. Surgical pathology showed a multifocal papillary TC with poorly differentiated component. He received a first RAI treatment two months after the surgery. Post treatment whole body scan showed uptake in the thyroid bed and upper mediastinum. A second cycle of RAI was given in 2001 (cumulative activity $370 \mathrm{mCi}$ ). Posttreatment scan was negative. In 2001 and in 2016 he had central compartment and bilateral neck dissection for recurrent disease. The first evidence of lung metastasis was in 2005. His metastatic disease had been observed for several years but in 2017, due to lung and liver progression, he was started on lenvatinib at a dose of $20 \mathrm{mg}$ daily. After 4 months with stable disease, lenvatinib was reduced to $20 \mathrm{mg}$ daily (5 days on, 2 off) because of toxicity (uncontrolled hypertension, mucositis, fatigue).

This unconventional treatment scheme was offered in accordance with patient preference and pharmacokinetic 

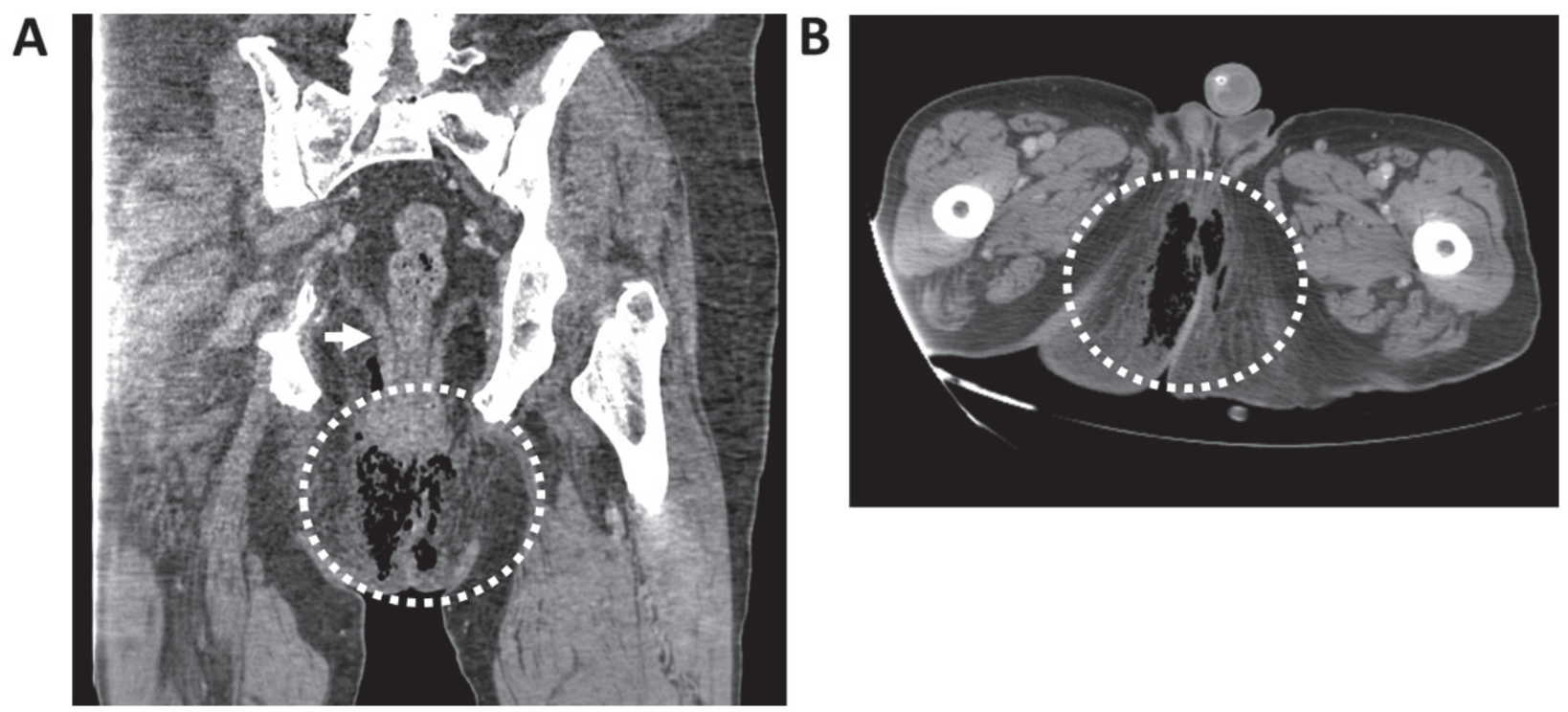

Figure 1. Radiologic features of Fournier's gangrene. (A) Coronal and (B) axial images of CT scan showing an abscess in the perianal area (white circles) with thickening of the levator ani muscle (arrow). Abscess tissue surrounded the anus and deepened caudally and posteriorly between the buttocks, and there was evidence of gaseous nuclei which were also involved in the anal, perineal and gluteus region, and extended anteriorly to the penile region, a presentation compatible with Fournier's gangrene.

data (half-life of lenvatinib: $28 \mathrm{~h}$ ) (9). It resulted in a better tolerability, less toxicity and increased adherence to therapy.

After 14 months of treatment with lenvatinib, he was admitted to the emergency room. He was hypotensive (90/55 $\mathrm{mmHg}$ ), tachycardic (150 beats per min), and afebrile. His hemoglobin level was $14.4 \mathrm{~g} / \mathrm{dl}$, with a hematocrit level of $42.5 \%$; white blood cell count was $24.9 \times 10^{3}$ cells $/ \mu$ l (with $87 \%$ neutrophils), and platelet count was $328 \times 10^{3}$ cells $/ \mu 1$. C-reactive protein was $26.33 \mathrm{mg} / \mathrm{dl}$, creatinine was $1.9 \mathrm{ng} / \mathrm{dl}$, serum sodium was $131 \mathrm{mmol} / 1$, serum potassium was $5.5 \mathrm{mmol} / \mathrm{l}$, and lactate level to venous blood gas was $1.79 \mathrm{mmol} / \mathrm{l}$. Fournier's gangrene severity index was 8 (10). Unfortunately, there was hemolysis in the sample.

At that time, other medications included levothyroxine, calcium, vitamin D3, silodosin, olmesartan, amlodipine, and sotalol. He was overweight (BMI: $28 \mathrm{~kg} / \mathrm{m}^{2}$ ) and the metabolic panel performed one month before the event showed dyslipidemia (triglycerides: $299 \mathrm{mg} / \mathrm{dl}$; LDL cholesterol: $107 \mathrm{mg} / \mathrm{dl}$; and HDL: $36 \mathrm{mg} / \mathrm{dl}$ ) with a fasting blood sugar of $88 \mathrm{mg} / \mathrm{dl}$.

An abdominal CT scan performed in the emergency room showed an abscess in the perianal area with thickening of the levator ani muscle. Abscess tissue surrounded the anus and deepened caudally and posteriorly between the buttocks, and there was evidence of gaseous nuclei which were also involved in the anal, perineal, and gluteus region and extended anteriorly to the penile region, a presentation compatible with FG (Fig. 1). No signs of progressive disease were found. Surgery was performed with an incision at the root of the scrotum, which was then washed and packed with iodoform gauze. As limitation of this report, blood and tissue culture data were not available. Empirical treatment with meropenem $1 \mathrm{~g}$, metronidazole $0.5 \mathrm{~g}$, and amoxicilline $2 \mathrm{~g}$ was started.

Clinical evolution was satisfactory and the patient was discharged after 7 days. Lenvatinib treatment was discontinued after the diagnosis of Fournier's gangrene. The patient experienced brain progression 6 months after lenvatinib was stopped. Because of the oligoprogression, stereotactic radiation therapy was administered without adding systemic treatment. Multiorgan progression occurred 8 months later. At that point the patient moved to another city. He was still alive 16 months after the event.

\section{Discussion}

FG is a type of necrotizing fasciitis that involves the perineal, perianal, and genital areas and most frequently affects elderly men. Traditional risk factors are diabetes, immunosuppression, recent surgery, and trauma to the genital and perianal area. Clinical manifestations usually present with acute and rapidly progressive edema, erythema, and tenderness. Advanced cases are defined by toxic symptoms including fever, tachycardia, hypotension, and septic shock. Clinical suspicion should be confirmed with surgical exploration. A CT scan can also help identify an infectious origin and delineate the extent of disease. Surgical debridement of the necrotic areas, along with parenteral broad-spectrum antibiotics, represents the gold standard of treatment. Early diagnosis and treatment are crucial for a better outcome, as the mortality of this condition is high (11).

Our patient developed FG with the only known risk factor being advanced age. No symptoms were reported in our chart that might have been suggestive of this condition. None of the available blood parameters measured prior to development of FG provided any indication about the development of FG.

To the best of our knowledge, this is the first reported case of FG in relation to lenvatinib treatment. According to the Naranjo Adverse Drug Reaction Probability Scale (12) and Jones algorithm (13), lenvatinib was determined to be the probable cause of FG (Tables SI and SII; http://www.pmidcalc. org/?sid=7249508\&newtest=Y).

In 2012, the FDA reported 22 cases of necrotizing fasciitis that developed during treatment of colorectal cancer with 
bevacizumab, with death resulting in $13.6 \%$ of patients (14). A safety alert regarding some cases related to bevacizumab has also been described in literature (15-17). Other cases have been reported in association with aflibercept for the treatment of colorectal cancer (18) and sunitinib in a patient treated for renal cell carcinoma (19). In all these cases, the condition developed in the absence of major risk factors other than antiangiogenic treatment. We assume that the condition is likely due to a perturbation of vascular endothelial cells of the skin due to the inhibition of VEGF/VEGFR signaling, since these drugs don't have other targets in common. It has been found that VEGF/VEGFR inhibition leads to a perturbation in the coagulation cascade, resulting in a prothrombotic state (20). Thrombosis in small skin vessels could lead to ischemia and necrosis of the tissue with secondary bacterial colonization, causing the development of the infection.

As regards the temporal relationship between the treatment and side effects, in our patient FG appeared 14 months after lenvatinib was started and was a late serious side effect. In previous reports $(15,16,18,19)$, the median time until the occurrence of this event was 6.5 months (range 4-12) and appeared during treatment with the antiangiogenic agent. In clinical practice, physicians worry about acute side effects, but late side effects could be serious as well and few data are available on this topic (14). Delayed or late onset adverse events are common with VEGF/VEGFR. For instance, proteinuria and nephrotic syndrome develop over time (21). FG, in previous reports as well as in our case, has been described after several months of therapy. So far, most studies have not been able to address late-onset toxicity incidence because treatment times in clinical trials are shorter. Post-marketing analyses help in identifying late and/or severe adverse events (14). Since TC is commonly a slow-growing cancer compared to other solid tumors and since the median progression-free survival associated with lenvatinib in this setting has been shown to be long (18 months) (5), it would not be surprising if other late-onset toxicities come to light in post-marketing studies or anecdotal cases.

The worldwide growing use of lenvatinib for the treatment of RAI refractory thyroid cancer and other tumors will lead the clinicians to deal with adverse events not reported in the SELECT study (5) or in the reports of real-life experience (22). Combining knowledge of the risk factors-signs of FG and our findings reported in this case, should push clinicians, during periodic evaluation of patients on lenvatinib, to ask questions about symptoms of discomfort of perineal area and consider anorectal abscess/FG in differential diagnosis. In particular, in patients with risk factors for FG and under treatment with antiangiogenic drugs, the choice of a more specific follow-up based on periodic and symptoms-based inspection of the genitalia, perineum and digital rectal examination should be considered. Moreover, a clinical evaluation of this body area may be appropriate prior to prescribing antiangiogenics.

In conclusion, this is the first case reported in literature of Fournier's gangrene likely related to lenvatinib treatment in a patient with advanced thyroid cancer. In our opinion, Fournier's gangrene is a class effect of antiangiogenic treatment. Clinicians who deal with these drugs in clinical practice should be aware of the possibility, even though remote, that this condition may develop given the fact that it has a high mortality rate and prompt diagnosis and management are needed.

\section{Acknowledgements}

Not applicable.

\section{Funding}

No funding was received.

\section{Availability of data and materials}

The datasets used and/or analyzed during the current study are available from the corresponding author on reasonable request.

\section{Authors' contributions}

CD, GG, VR and RF evaluated the patient and participated in treatment planning. TG evaluated radiological images and generated Fig. 1. MB and RF wrote the manuscript. All authors read and approved the final manuscript.

\section{Ethics approval and consent to participate}

Not applicable.

\section{Patient consent for publication}

Informed consent was provided by the patient for the publication of his clinical information and images.

\section{Competing interests}

CD declares receiving an Advisory Board fee from EISAI. $\mathrm{MB}, \mathrm{GG}, \mathrm{VR}, \mathrm{TG}$ and RF declare that they have no competing interests.

\section{References}

1. Lamartina L, Grani G, Durante C and Filetti S: Recent advances in managing differentiated thyroid cancer. F1000Res 7: 86, 2018.

2. Kudo M, Finn RS, Qin S, Han KH, Ikeda K, Piscaglia F, Baron A, Park JW, Han G, Jassem J, et al: Lenvatinib versus sorafenib in first-line treatment of patients with unresectable hepatocellular carcinoma: A randomised phase 3 non-inferiority trial. Lancet 391: 1163-1173, 2018

3. Motzer RJ, Hutson TE, Glen H, Michaelson MD, Molina A, Eisen T, Jassem J, Zolnierek J, Maroto JP, Mellado B, et al: Lenvatinib, everolimus, and the combination in patients with metastatic renal cell carcinoma: A randomised, phase 2, open-label, multicentre trial. Lancet Oncol 16: 1473-1482, 2015.

4. Fala L: Lenvima (Lenvatinib), a multireceptor tyrosine kinase inhibitor, approved by the FDA for the treatment of patients with differentiated thyroid cancer. Am Health Drug Benefits 8 (Spec Feature): 176-179, 2015

5. Schlumberger M, Tahara M, Wirth LJ, Robinson B, Brose MS Elisei R, Habra MA, Newbold K, Shah MH, Hoff AO, et al: Lenvatinib versus placebo in radioiodine-refractory thyroid cancer. N Engl J Med 372: 621-630, 2015.

6. Kim SY, Kim SM, Chang H, Kim BW, Lee YS, Chang HS and Park CS: Safety of tyrosine kinase inhibitors in patients with differentiated thyroid cancer: Real-world use of lenvatinib and sorafenib in Korea. Front Endocrinol (Lausanne) 10: 384, 2019.

7. Krajewska J, Kukulska A and Jarzab B: Drug safety evaluation of lenvatinib for thyroid cancer. Expert Opin Drug Saf 14: 1935-1943, 2015. 
8. Schmidinger M: Understanding and managing toxicities of vascular endothelial growth factor (VEGF) inhibitors. EJC (Suppl 11): 172-191, 2013.

9. Capozzi M, De Divitiis C, Ottaiano A, von Arx C, Scala S, Tatangelo F, Delrio P and Tafuto S: Lenvatinib, a molecule with versatile application: From preclinical evidence to future development in anti-cancer treatment. Cancer Manag Res 11: 3847-3860, 2019

10. Laor E, Palmer LS, Tolia BM, Reid RE and Winter HI: Outcome prediction in patients with Fournier's gangrene. J Urol 154 89-92, 1995.

11. Singh A, Ahmed K, Aydin A, Khan MS and Dasgupta P: Fournier's gangrene. A clinical review. Arch Ital Urol Androl 88 : 157-164, 2016

12. Naranjo CA, Busto U, Sellers EM, Sandor P, Ruiz I, Roberts EA, Janecek E, Domecq C and Greenblatt DJ: A method for estimating the probability of adverse drug reactions. Clin Pharmacol Ther 30: 239-245, 1981

13. Jones JK: Adverse drug reactions in the community health setting: Approaches to recognizing, counseling, and reporting. Fam Community Health 5: 58-67, 1982.

14. Shamloo BK, Chhabra P, Freedman AN, Potosky A, Malin J and Weiss Smith S: Novel adverse events of bevacizumab in the US FDA adverse event reporting system database: A disproportionality analysis. Drug Saf 35: 507-518, 2012.

15. Gamboa EO, Rehmus EH and Haller N: Fournier's gangrene as a possible side effect of bevacizumab therapy for resected colorectal cancer. Clin Colorectal Cancer 9: 55-58, 2010.

16. Sendur MA, Aksoy S, Özdemir NY and Zengin N: Necrotizing fasciitis secondary to bevacizumab treatment for metastatic rectal adenocarcinoma. Indian J Pharmacol 46: 125-126, 2014.
17. Ugai T, Norizuki M, Mikawa T, Ohji G and Yaegashi M: Necrotizing fasciitis caused by Haemophilus influenzae type $b$ in a patient with rectal cancer treated with combined bevacizumab and chemotherapy: A case report. BMC Infect Dis 14: 198, 2014

18. Gonzaga-López A, Muñoz-Rodriguez J and Ruiz-Casado A. Necrotising fasciitis in a patient treated with FOLFIRI-aflibercept for colorectal cancer: A case report. Ann R Coll Surg Engl 99: e225-e226, 2017.

19. Piszczek J, Dalton B, Peters T, Ruether D and Urbanski S Extensive necrotizing fasciitis associated with sunitinib therapy. Clin Genitourin Cancer 12: e47-e49, 2014.

20. Kuenen BC: Analysis of prothrombotic mechanisms and endothelial perturbation during treatment with angiogenesis inhibitors. Pathophysiol Haemost Thromb 33 (Suppl 1): S13-S14, 2003.

21. Moriconi D, Cappagli V, Viola D, Molinaro E, Agate L, Lorusso L, Egidi MF and Elisei R: Proteinuria is a late onset adverse event in patients treated with cabozantinib: A single center experience. Nephrol Dial Transpl 33: i388-i38918, 2018.

22. Locati LD, Piovesan A, Durante C, Bregni M, Castagna MG, Zovato S, Giusti M, Ibrahim T, Puxeddu E, Fedele G, et al: Real-world efficacy and safety of lenvatinib: Data from a compassionate use in the treatment of radioactive iodine-refractory differentiated thyroid cancer patients in Italy. Eur J Cancer 118: 35-40, 2019. 